Diabetologe 2018 $\cdot 14: 530-531$

https://doi.org/10.1007/s11428-018-0415-y

(c) Springer Medizin Verlag $\mathrm{GmbH}$, ein Teil von Springer Nature 2018

CrossMark

\title{
Hans-Peter Hammes
}

5. Medizinische Klinik, Sektion Endokrinologie, Universitätsmedizin Mannheim, Mannheim, Deutschland

\section{Warum über diabetische Retinopathie schreiben?}

tiven Antidiabetika neue Erkenntnisse ergaben und

wurde in der Vergangenheit erkennbar in den Hintergrund gedrängt. Für das nachlassende Interesse an dieser Thematik, das bei den Besuchen der Symposien der AGDA (Arbeitsgemeinschaft Diabetes und Auge) oder der Postersessions $\mathrm{zu}$ diesem Thema auf dem Jahreskongress unserer Fachgesellschaft oder der europäischen Diabetesgesellschaft am deutlichsten wird, gibt es vielfältige und nachvollziehbare Gründe: Zum einen scheint das Problem Erblindung durch die ophthalmologische Therapie gelöst worden $\mathrm{zu}$ sein, zum anderen verminderte eine ausschließlich von Ophthalmologen durchführbare Behandlungsmaßnahme - die intravitreale ophthalmologische Medikation - das Interesse auf diabetologischer Seite. Eine interdisziplinäre Herausforderung ist und bleibt allerdings die gegenseitige Information über die diabetologischen Eckdaten der Patienten einerseits und, von ophthalmologischer Seite, das Retinopathiestadium und die vorgeschlagenen Therapiekonzepte andererseits. Dabei sind diese gegenseitigen Auskünfte Grundlage einer bestmöglichen und v. a. kosteneffektiven Versorgung der Betroffenen.

Aktuell gibt es genügend Gründe, das Thema diabetische Retinopathie auf die Tagesordnung zu setzen:

- weil viel Wissen um die Pathogenese und das Erscheinungsbild der diabetischen Retinopathie in der Diabetologie verloren ging,

- weil die Funktion der Retinopathie im Kontext der Systemerkrankung Diabetes vernachlässigt wird,

- weil sich in jüngster Zeit durch die Nebenwirkungsprofile der hocheffek-
- weil viele definierte Barrieren existieren, die eine ophthalmologische Untersuchung erschweren.

Das Autorenteam, das sich dem Leitthema diabetische Retinopathie in dieser Ausgabe widmet, stellte sich u. a. folgende Fragen:

- Ist die Retinopathie wirklich seltener geworden, d.h. sind rückläufigen Untersuchungszahlen tatsächlich trotz ansteigender Patientenzahlen gerechtfertigt?

- Hat die diabetologische Behandlung mit Fokus allein auf den Blutzuckerspiegel den Stellenwert, der ihr zugeschrieben wird?

- Kann die Injektion ins Auge Laserbehandlungen auf Dauer ersetzen?

Margerete Voigt und Mitautoren widmen sich dem Kapitel der Epidemiologie der Retinopathie unter differenzierter Betrachtung verschiedener Versorgungsstufen.

\section{》) Die klinische Stadien- einteilung ist wesentliche Grundlage der interdisziplinären Kommunikation}

Hansjürgen Agostini und Focke Ziemssen rücken aus ophthalmologischer Sicht die Pathomechanismen der gestörten Schrankenfunktion diabetischer Gefäße im Auge und der Neurodegeneration als neuem Element der Retinopathie in den Vordergrund.

Die klinische Stadieneinteilung als wesentliche Grundlage der interdiszipli- nären Kommunikation ist ein wichtiges Kernstück des Themas und Pflichtlektüre eines jeden angehenden Diabetologen DDG (Deutsche Diabetes Gesellschaft) und wurde, ebenso wie das Kapitel über die etablierten und neuen Techniken der Bildgebung, von Focke Ziemssen und Mitautoren aufbereitet. Sie berichten über Weitwinkelsysteme, die ohne Mehrfelderfotografie mehr Retinopathie erfassen, über den unbestrittenen Stellenwert optischer Kohärenztomographien und über die noch experimentelle, aber beeindruckende Technik der OCTAngiographie (OCT: optische Kohärenztomographie), die in Zukunft ihren Platz in der Bildgebung bei Patienten mit diabetischer Retinopathie definieren wird. Automatische retinale Bildanalysealgorithmen (ARIAS) halten zwangsläufig Einzug in die diabetologische Anwendung und werden vorgestellt.

Die Thematik wird durch 3 therapeutische Kapitel abgerundet: Der Beitrag zur diabetologischen Therapie befasst sich v. a. mit der Effektstärke von antihyperglykämischen und antihypertensiven Konzepten und ausführlicher mit der Interpretation der neuen Daten zur Retinopathieentstehung bei GLP-1Rezeptor-Agonisten (GLP-1: „glucagonlike peptide 1").

Dem großen Thema der Behandlung des diabetischen Makulaödems widmet sich Georg Spital, insbesondere dem Aspekt der Effektstärke der IVOM (intravitreale operative Medikamentengabe) im Praxisalltag.

Diabetologisches Grundwissen beinhaltet, dass die panretinale Laserkoagulation Erblindung verhindert. Möglicherweise kann diese zerstörende Therapie bald durch bessere Konzepte ersetzt 
werden, wie von Norbert Bornfeld ausführlich dargelegt.

Ich wünsche Ihnen bei der Lektüre des Themenheftes diabetische Retinopathie viele Neuigkeiten. Bleiben Sie interessiert.

Mit freundlichen kollegialen Grüßen

Ihr

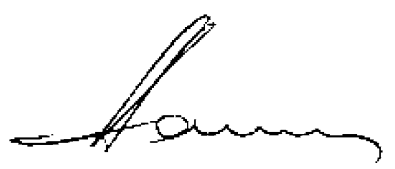

Hans-Peter Hammes, Mannheim

\section{Korrespondenzadresse}

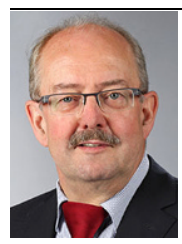

Prof. Dr. med. Hans-

Peter Hammes

5. Medizinische Klinik,

Sektion Endokrinologie,

Universitätsmedizin

Mannheim

Theodor-Kutzer-Ufer

1-3, 68167 Mannheim,

Deutschland

hp.hammes@umm.de

Interessenkonflikt. H.-P. Hammes weist auf folgende Beziehungen hin: Beratung: Novo Nordisk, MSD; Vortragstätigkeit: Novartis, Bayer, Sanofi, Heidelberg Engineering.

\section{Oskar Medizin-Preis 2018 zum Thema “Adipositas" verliehen}

Der Oskar Medizin-Preis zählt zu den renommiertesten und mit $\mathbf{5 0 . 0 0 0 ~ E u r o ~}$ zu den höchstdotierten Auszeichnungen für Ärzte in Deutschland. Er würdigt in diesem Jahr herrvorragende wissenschaftliche Arbeiten und medizinische Leistungen auf dem Gebiet der Kankheit „Adipositas“. Die Jury entschied sich zur Vergabe des Preises an Herrn PD Dr. Felix Nickel (Universität Heidelberg) für seine wissenschaftliche Arbeiten im Bereich der effektiven und nachhaltigen Behandlung der Adipositas. Der Preis wurde am 5. November 2018 in der URANIA in Berlin von der Stiftung Oskar-Helene-Heim verliehen.

Die prämierte Arbeit belegt die positiven Effekte der Adipositaschirurgie Herr PD Dr. Felix Nickel hat in verschiedenen Studien gezeigt, dass die Adipositatschirurgie neben einer signifikanten Reduktion des Gewichtes eine positive Auswirkung auf die Lebenserwartung, die Lebensqualität, das Körperbild und das allgemeine Selbstvertrauen hat. Es spielte dabei keine Rolle, ob die Patienten mittels Schlauchmagen oder Magenbypass operiert wurden. Zudem verbesserte sich die gastrointestinale Lebensqualität und diverse Nebenerkrankungen des Übergewichts. Die Patienten wurden anhand verschiedener validierter Fragebögen prospektiv befragt. Die Studien belegen, dass die Adipositaschrirurgie bei Versagen konservativer Maßnahmen unbedingt immer als Therapiekonzept in Betracht zu ziehen ist. Das Preisgeld investiert Herr PD Dr. Felix Nickel in weitere Studien, die z.B. den Einfluss verschiedener bariatrischen Chirurgieverfahren auf die Leber analysieren.

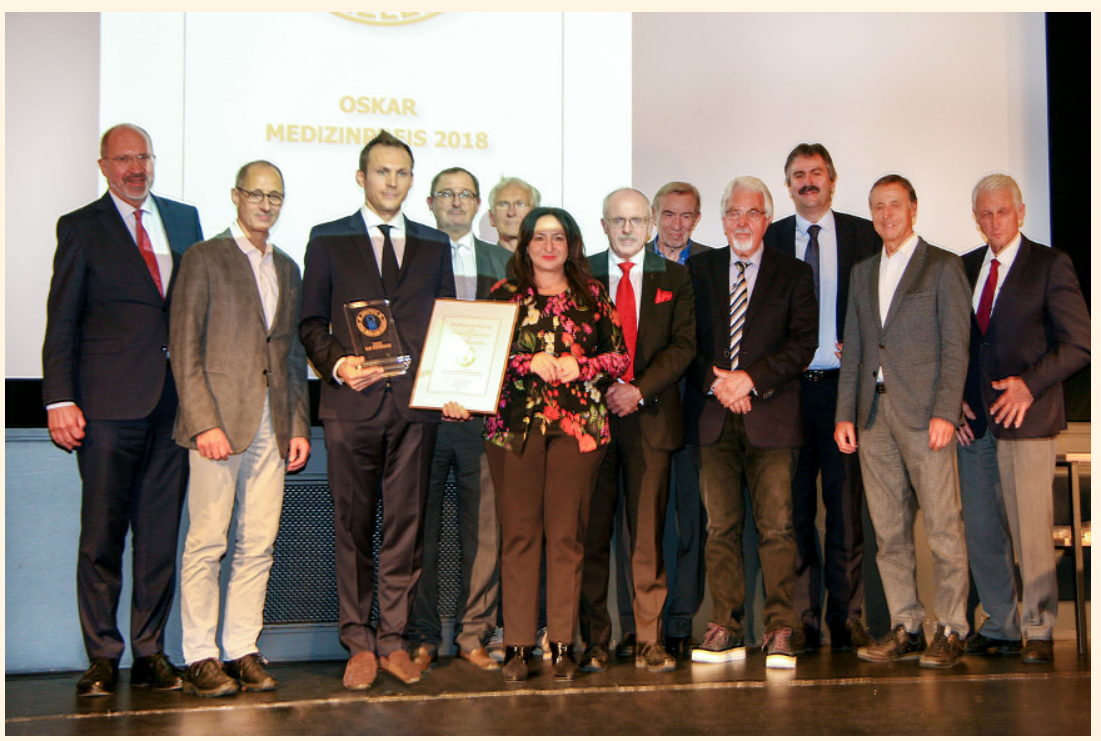

v.I.n.r.: Prof. Dr. W. Kuhla, Vorsitzender der Jury und des Stiftungskuratoriums; Prof. Dr. A. Pfeiffer, Mitglied der Jury; PD Dr. F. Nickel, Preisträger; Dr. G. Jonitz, Präsident der Ärztekammer; Prof. Dr. S. Müller-Lissner, Mitglied der Jury und des Stiftungskuratoriums; D. Kolat, Senatorin für Gesundheit, Pflege und Gleichstellung; Prof. Dr. D. Birk, Laudator und Mitglied der Jury; Prof. Dr. R. Loddenkemper, Mitglied des Stiftungskuratoriums; W. Ukas, Stellvertretender Vorsitzender des Stiftungskuratoriums; Prof. Dr. C. Perka, Mitglied des Stiftungskuratoriums; Prof. Dr. W. Noack, Mitglied des Stiftungskuratoriums; T. M. Höhn, Geschäftsführer der Stiftung

\section{Die Stiftung Oskar-Helene-Heim}

Die Stiftung Oskar-Helene-Heim in Berlin fördert Wissenschaft und Forschung in der Medizin. Die Stiftung unterstützt Forschungsprojekte und gemeinnützige gesundheitsfördernde Vorhaben und verleiht Stipendien. Sie vergibt jährlich den Oskar Medizin-Preis und die HeleneMedaille. Die Stiftung Oskar-Helene-Heim wurde 1905 von Oskar Pintsch, seiner Ehefrau Helene und dem Arzt Dr. Konrad Biesalski gegründet, mit dem Ziel gesundheitliche Handicaps junger Menschen zu heilen und zu überwachen.

Quelle: Stiftung Oskar-Helene-Heim, www.stiftung-ohh.de 